\title{
"LA HISTORIA CLÍNICA COMO PRUEBA PARA LA RESPONSABILIDAD CIVIL POR MALA PRÁCTICA MÉDICA"
}

\section{Beatriz Gallegos Pérez ${ }^{1}$}

\section{Resumen}

Para acreditar la responsabilidad civil de los médicos, cuando se encuentren demandados por que se les atribuye un daño, adquiere gran importancia el acto médico. Es considerada como el instrumento legal donde se muestra la actuación del médico constituyéndose como la prueba documental de mayor peso jurídico ante casos de reclamos de tipo civil.

Para el desarrollo del tema tomaremos como hipotesis el analizar las etapas del acto médico en forma continua, permitirá conocer los elementos característicos de la historia clinica que se han incumplido en la lex artis ad hoc, para determinar la responsabilidad civil del médico al ser utilizada como prueba documental.

Utilizando para el desarrollo, los métodos contemporáneos de investigacion como lo son la doctrina analítica y la sociología jurídica.

Lo anterior justifica realizar un analisis a la doctrina jurídica y la normativa para ser considerada como prueba en el proceso de responsabilidad civil médica.

Palabras clave: Acto médico, Expediente Clínico, Responsabilidad Médica, Lex artis ad hoc, Proceso Civil.

\section{INTRODUCCION}

En México, para la atencion médica es importante la historia clínica, la ausencia o deficiencia de este documento, produce una presunción contra aquellos médicos que han realizado la atencion médica del paciente,

\footnotetext{
${ }^{1}$ Estudiante del quinto semestre en el Doctorado en Estudios Jurídicos del PNPC de la División Académica de Ciencias Sociales y Humanidades en la Universidad Juárez Autónoma de Tabasco. E-mail: Beatrizgallegos12@hotmail.com
} 
en caso de producirse la existencia de un posible daño por mala práctica médica, y de existir ausencia o deficiencia de la historia clínica puede generar un perjuicio para aquellos médicos que por ley están obligados a su elaboración y manifestar de forma escrita toda la informacion necesaria para ciencia médica, procediendo a documentar de forma detallada todo el curos del acto médico.

Es decir que el hecho que una historia clinica se encuentre de forma incompleta o deficiente al ser realizada por el personal médico, genera un riesgo innecesario para el derecho a la vida y a la salud de los pacientes, riesgo que no debe ser justificado por el riesgo que conlleva la práctica del ejercicio de la medicina. (TESIS: 2002569. 1a.XXVIII/2013, 10a.).

Con esta práctica se producen varios efectos negativos en la prestación del servicio a los pacientes, considerando que la información comprendida en un expediente clínico va a generar dos condiciones.

La primera va a dar las facilidades para que profesional de la salud realice un seguimiento acertado y diligente en referencia al problema de salud que presenta el paciente.

La segunda proporciona al paciente, como a sus familiares o persona que está autorizada las herramientas necesarias para que en base a la informacion relacionada con sus padecimientos o condiciones de salud se tomen las decisiones correctas.

Por consiguiente la inexistencia o insuficiencia de la historia clínica va a generar consecuencias en contra del médico, el cual tendrá las siguientes desventajas:

1. Reconocerá la verdad de los hechos que sean afirmados por el demandante.

2. Significa una sospecha que no existe interés del médico en colaborar en el proceso judicial.

3. Al generarse la motivación de la inversion de la carga de la prueba, será el médico quien tendrá que probar que su actuación fue diligente, y con una historia clínica insuficiente o inexistente podría ser mucho más difícil. (TESIS: 2002569. 1a. XXVIII/2013, 10a.).

Es evidente que será el demandante quien debe probar como principio básico la culpa del médico, para ello deberá demostrar su impericia, imprudencia, negligencia o cualquier otro deber que se haya incumplido en la atencion médica. De acuerdo con la doctrina moderna existe la teoria llamada de pruebas dinámicas, donde el medico inculpado debe demostrar que no ha tenido culpa en su actuación profesional, para eso deberá aportar los elementos que demuestren su inocencia.

Contrariamente a lo que se ha expresado el hecho de que una historia clínica se encuentre mal confeccionada será una prueba objetiva y suficiente para asignar responsabilidad penal o civil a los profesionales de la salud, así como en caso de encontrarse la historia clínica correctamente elaborada no se podrá suponer que el paciente tuvo una atencion correcta y de calidad. (TESIS: 2002569. 1a. XXVIII/2013, 10a.).

Lo anterior ha generado la siguiente pregunta general: 
¿Se puede considerar la historia clínica cómo medio de prueba para determinar la responsabilidad civil médica en casos de mala práctica?

Misma que ha generado los siguientes cuestionamientos:

¿Cuáles son las características y la estructura de la historia clínica?

¿Cuáles son las normas mexicanas que regulan la historia clínica?

¿Cuál es la importancia de la obligatoriedad del consentimiento informado para ser parte en la historia clínica?

¿En qué momento del proceso civil se determina que la historia clínica puede ser utilizada como prueba de la mala práctica médica?

Con referencia a La Lex Artis médica o estado del arte médico, se ha determinado que es el conjunto de normas o criterios valorativos que el médico, en posesión de conocimientos, habilidades y destrezas, deberá utilizar de manera cuidadosa en el caso concreto de un enfermo, donde tales procedimientos han sido universalmente aceptados por otros médicos. (TESIS: 2004786. I.4o.A.91 A 10a.).

\section{EL ACTO MÉDICO}

La historia clínica establece el relato patográfico o biografía patológica del paciente, es decir que en ella se expresará la relación médico - paciente, por lo que le permite tener un valor fundamental, tanto en el ámbito clínico como también a la hora de juzgar las acciones de los profesionales de la medicina. Por ello la ausencia o deficiencia de la historia clínica, produce una presunción en contra de los médicos que trataron al paciente. (TESIS: 1a.XXVIII/2013 (10a), Aislada Civil, núm. 2002569)

Para acreditar la responsabilidad civil de los médicos, cuando se encuentren demandados por que se les atribuye un daño, serán ellos quienes tengan que probar su debida diligencia, uno de los elementos de la responsabilidad civil extracontractual, la culpa, por otro lado el demandante tiene que acreditar los otros elementos de la responsabilidad civil extracontractual, los cuales son: daño y nexo causal, es decir cuando el paciente alegue que un médico le ocasiono un daño, se actualiza lo que se ha denominado reinversión de la carga de la prueba a favor de la actora en el juicio. Donde al médico le corresponde acreditar su debida diligencia en la debida atencion médica del paciente que argumenta haber sufrido un daño. (TESIS: 1ª CCXXVII72016, 10a, Aislada Civil, núm. 2012513)

El acto médico necesita de ser documentado gráficamente, y para ello es en la historia clinica donde se debe plasmar, conocida también como ficha clínica, historial clínico o expediente clínico. En ella se registrarán de manera ordenada los datos objetivos y los subjetivos del paciente, también se registrarán acontecimientos 
médicos de importancia que se hayan generado durante la atención médica, por lo tanto se puede considerar como una herramienta universal para llevar a efecto el cuidado del paciente. Por tal motivo es importante hacer su elaboración de la manera más pulcra, ya que es considerada como el instrumento legal donde se muestra la actuación del médico constituyéndose como la prueba documental de mayor peso jurídico ante casos de reclamos de tipo penal, civil o administrativo. Lo anterior justifica realizar un analisis a la doctrina jurídica y la normativa que regula la historia clínica en México para ser considerada como prueba en el proceso de responsabilidad civil médica. (Fidel Herminio, 2009.)

\section{LA HISTORIA CLINICA SUS CARACTERISTICAS Y SU ESTRUCTURA}

\section{Concepto}

Para que se pueda materializar el derecho a la protección de la salud, es de gran importancia que se considere al expediente clínico como un instrumento para poder determinar las actuaciones del profesional de la salud. Se le considera como el único conjunto de información y datos personales de un paciente, mismos que deberán estar contenidos en otros documentos ya sean escritos, gráficos, imagenológicos, electrónicos, magnéticos, electromagnéticos, ópticos, magneto-ópticos, así como aquellos que se realicen con otras tecnologías, en las cuales mediantes ellas se hagan constar la atención médica en los diferentes momentos de su procedimiento, así como las deferentes intervenciones que realice el personal del área de salud, donde describirán el estado de salud del paciente; debiendo incluir en su caso, todos los datos e información que esté relacionada con el bienestar físico, mental y social del paciente. (Norma Oficial Mexicana NOM-004-SSA3-2012, Del expediente clínico.)

Tomando como fundamento su contenido y que no tan sólo integra una exposición sistemática de acontecimientos clínicos pasado y presentes, porque además reúne información de diferentes tipos y de la más variada, a la historia clínica se le ha llegado a definir como expediente clínico. La historia clínica tiene como principal función la asistencia, pues es a través de ella que se puede otorgar una atención continua a los pacientes por el personal médico. Como también permite la docencia cuando es utilizada en la docencia, realización de estudios de investigación y epidemiología, la evaluación de calidad asistencial, la planificación y gestión de salud, pero también es importante mencionar que se utiliza en casos legales de alguna situación jurídica que esté relacionado con el médico tratante o cualquier otra donde se requiera. (José Manuel, 2013.) 


\section{Características}

La institución médica será la propietaria del expediente clínico la cual tendrá de forma obligatoria conservarla como un mínimo de hasta de cinco años, que serán contados a partir de la fecha en que se produjo el último acto médico, tomando como base lo esencial del expediente clínico este debe ser de la siguiente manera.

A. Confidencialidad: Para poder integrar el expediente clínico es necesario que el paciente facilite al profesional de salud información personal para efectuar el proceso asistencial de manera certera y precisa. Para ello el paciente cuenta con la seguridad que dicha informacion no podrá hacerse pública o proporcionarse a persona no autorizada por el paciente, se entiende que existe un acuerdo de tipo moral al silencio y que también lo considera la ley denominado como el secreto profesional. (José Manuel, 2013.)

B. Seguridad: Es importante poder identificar al paciente así como los médicos que han intervenido en todo el proceso de la atención médica.

C. Disponibilidad: Tomando en consideración que el expediente clínico es un documento confidencial, deberá ser disponible cuando se requiera para facilitar los procesos jurídicos en los términos que especifique la ley. (Lineamientos para el manejo del Expediente Clínico, Hospital Juárez de México, 2014.)

D. Intransferible: Debe realizarse únicamente para un paciente determinado, considerando que se dará seguimiento en el proceso salud-enfermedad, agregando datos que puedan identificar al paciente de manera clara.

E. Legibilidad: La falta de claridad en la escritura es un defecto muy concurrido por los médicos al realizar el expediente clínico, lo que ocasiona complicaciones para quien trata de interpretar los manuscritos, n o tan solo para los profesionales médicos, también complica la interpretación de aquellos que requieran de la utilidad de su informacion. Para una mayor comprensión se deberán evitar las abreviaturas, los médicos deberán escribir su nombre y su firma y de ser posible imponer un sello. (Fernando Guzmán, Scielo)

F. Objetiva y veraz: Deberá estar basada en hechos reales que describan la situación de salud del paciente y no como el médico suponga que debe ser.

G. Irreemplazable: Su escritura y redacción no debe ser reemplazada por otra forma tal como la memoria de los médicos o enfermeras, debido a la gran informacion que se genera con los estudios especializados y aplicación de medicamentos que no es posible recordar todo, en cuanto a que otro médico tome el procedimiento debe tener la informacion más precisa de lo que se le ha estado aplicando al paciente para dar continuidad.

H. Completo y con rigor técnico: La informacion que se recabe estará realizada con criterios objetivos y científicos, así como aquellos datos que se registren sobre la enfermedad del paciente deben reflejar que se ha sometido a todas las etapas medicas - legales que se requieren para el acto clínico - asistencial, como lo determina 
la Norma Oficial Mexicana del Expediente Clínico. (Maritza Agüero, 2010)

\section{Estructura}

El contenido de los expedientes clínicos puede variar dependiendo del lugar en el que se realice la atención médica, para ello la Norma Oficial Mexicana 168, ha establecido tres tipos de expedientes clínicos de acuerdo a su contenido y su estructura, sin ninguna excusa todo expediente clínico deberá contener los datos generales del paciente y de la institución que otorga el servicio médico: Tipo, nombre y domicilio del establecimiento, así como el nombre de la institución a que pertenece, con relación al paciente se tomara el nombre, sexo, edad, domicilio y ocupación del usuario. (Nicolás Bañuelos, Metabase)

Tipos de expediente clínicos:

1. El expediente clínico en el servicio de urgencias: Aquellos servicios que se realicen en el área de urgencias quedaran exentos de la integración dl expediente clínico solo realizarán un registro en una nota inicial con la finalidad de dar agilidad a la atención médica, en la cual de manera concreta se anotaran los datos positivos y los datos negativos que puedan ser útiles para elaborar un diagnóstico y poder dar un tratamiento y pronóstico de la situación actual del paciente, previamente será identificado, se anotará la fecha, hora lugar y nombre con las firmas de los médicos que reciben y dan la atención médica.

2. El expediente clínico en el servicio de consulta externa: Es el servicio de la atencion médica realizada por los médicos especialistas, para aquellos pacientes ambulatorios que requieran de alguna de las cuatro especialidades médicas básicas, así como de cualquier otra subespecialidad, y consiste en realizar un interrogatorio, exploración física para poder determinar un diagnóstico. (NORMA Oficial Mexicana NOM-040-SSA2-2004, En materia de información en salud.)

3. El expediente clínico en el servicio de hospitalización.: Se realizará el registro de la atención integral que se haya realizado a un adulto enfermo, que tendrá como objetivo el diagnóstico y el tratamiento que sea de tipo quirúrgico de las enfermedades que afectan los órganos, los sistemas internos y se buscará su prevención. (NORMA Oficial Mexicana NOM-040-SSA2-2004, En materia de información en salud.)

Si el paciente llegase a requerir consulta externa o una hospitalizacion, su expediente clínico deberá estar integrado por los siguientes documentos:

1. Historia clínica: Documento de gran importancia, de allí se obtiene la información que se utilizará para elaborar un juicio clinico, es el primer documento que se elabora para la atencion medica del paciente, para que se considere como una historia clínica apropiada deberá contar con la característica de ser un documento redactado en forma descriptiva. 
2. Nota inicial o de ingreso o de revisión: Documento donde se realiza la interpretación de la informacion que se ha descrito en la historia clínica, siendo en esta etapa donde a base de juicio o ejercicio clínico el médico tomara los datos positivos negativos que conducirán para realizar los diferentes diagnósticos que sustentarán el plan de estudio y el tratamiento para llegar a un pronóstico del estado clinico de la salud del paciente. (Fidel Herminio, 2010:71 y 72 )

3. Nota de evolución: Debe ser un documento de fácil ubicación, se deberá anotar de manera exacta el momento en que ha sido realizado y la identificación completa y precisa de quien lo ha elaborado, debiendo exponer los cambios y reacciones que se consideren importantes que ha tenido el paciente en comparación a su anterior revisión médica, debiendo sustentar los cambios terapéuticos que se vayan a generar a partir de la revisión siempre bajo las direcciones que manifiesta la Lex Artis ad hoc. (Fidel Herminio, 2010:71 y 72 )

4. Notas de egresos: Su elaboración estará a cargo del médico es considerada como un resumen de todo el procedimiento médico realizado en el tiempo que se encontró hospitalizado el paciente, su contenido será el siguiente:

Fecha de ingreso/egreso, Motivo del egreso, diagnósticos finales; resumen de la evolución y el estado actual, manejo durante la estancia hospitalaria, problemas clínicos pendientes, plan de manejo y tratamiento, recomendaciones para vigilancia ambulatoria, atención de factores de riesgo (incluido abuso y dependencia del tabaco, del alcohol y de otras sustancias psicoactivas), pronóstico, en caso de defunción, señalar las causas de la muerte acorde a la información contenida en el certificado de defunción y en su caso, si se solicitó y se llevó a cabo estudio de necropsia hospitalaria. (Norma Oficial Mexicana NOM-004-SSA3-2012, Del expediente clínico.)

5. Notas de evaluación pre anestésico: Procedimiento donde se efectuará la descripción de cuál será la técnica quirúrgica que se utilizará y el reporte de las indicaciones de la anestesia, en aquellos procedimientos donde participe un médico anestesiólogo sus reportes e indicaciones deberán formar parte de la historia clínica, el contenido se efectuará de manera exhaustiva con todos los detalles del procedimiento.

Es importante mencionar el trabajo del médico anestesista, que realiza dentro de la práctica quirúrgica, teniendo las mismas consecuencias y responsabilidades en su trabajo que el médico cirujano que es el jefe del equipo.

6. Nota preoperatoria: La realizará el cirujano que tenga a cargo la intervención del paciente, debiendo contener como mínimo la fecha de la cirugía, diagnostico, plan quirúrgico, tipo de intervención quirúrgica, riesgo quirúrgico, los cuidados y las indicaciones preoperatorias y el pronóstico. (Norma Oficial Mexicana NOM-004SSA3-2012, Del expediente clínico)

7. Nota postoperatoria: Forma un extracto de la cirugía practicada debiendo contener el diagnostico preoperatorio, el plan de la operación, el tipo de operación realizada, un diagnostico postoperatorio, descripción 
de la técnica quirúrgica, los hallazgos transoperatorios, un reporte de gasas y de las compresas, así como incidentes y accidentes, una cuantificación de sangrado, la realización de estudios de servicios auxiliares de diagnósticos, cuál será el tratamiento transoperatorio, los datos del personal que participo, el estado postquirúrgico inmediato, los pronósticos, el envío de piezas a biopsia u otros hallazgos considerados como importantes. (Francisco Hernández, 2012.)

8. Notas de enfermería: Aquellos que presten el servicio de enfermería deberán mantener la relación informativa que existe entre el médico y el personal de enfermería, siendo estos quienes están más tiempo con el paciente, sus observaciones tienen gran importancia y deben ser registradas puntualmente en la respectiva, como también deberán describir las acciones realizadas durante su turno laboral, como lo son la aplicación de medicamentos, signos vitales o reacciones inesperadas del paciente. (Fidel Herminio, 2010)

9. Reporte de paraclínicos realizados: Son aquellas pruebas que servirán de apoyo para los diagnósticos, los cuales su objetivo es aclarar las dudas o comprobar un diagnóstico previo, con ellos se tomarán las decisiones y evaluaciones por parte del médico para determinar un tratamiento específico que procure mejorar las condiciones de salud del paciente. Estos reportes, estudios o pruebas son: Audiometría, Visiometría, Optometría, Espirometría, Electrocardiogramas, Pruebas de imágenes diagnósticas, Rayos X de Tórax, Rayos X de columna dorso-lumbar, Pruebas de laboratorio clínico, entre otros de tipo especializados. (Exámenes Paraclínicos - Servicios Médicos Olimpus)

10. Carta de consentimiento bajo información: Es un documento donde el paciente, su representante legal o el familiar más cercano, expresan su aceptación a los procedimientos médico o quirúrgicos que tendrán como objetivo los diagnósticos terapéuticos, rehabilitadores, paliativos o en ocasiones para investigacion, generando informacion de los riesgos y beneficios para el paciente. (Norma Oficial Mexicana NOM-004-SSA32012, Del expediente clínico.)

11. Otros: Documentos de importancia en caso de fallecimiento, como el aviso al Ministerio Público, las responsivas médicas, notificaciones, etc. (Norma Oficial Mexicana NOM-004-SSA3-2012, Del expediente clínico.)

\section{LAS NORMAS MEXICANAS QUE REGULAN LA HISTORIA CLÍNICA}

\section{Ley General de Salud}

La Ley General de Salud expresa que el expediente clinico tiene dos alcances, el primero como una pequeña obligación relacionada con el principio de calidad en el servicio, cuando al referirse que la acreditación de 
la calidad de los servicios que se presten deben tomar en cuenta, la integración de expedientes clínicos entre otros más.

El segundo cuando manifiesta que los beneficiarios del Sistema de Protección Social en Salud tienen el derecho de contar con su expediente clínico. (LEY GENERAL DE SALUD, 2018: art. 77)

Así mismo el derecho al acceso a la información con referente a la salud del paciente se encuentra relacionado al expediente clínico de las personas. Las cuales tendrán el derecho de recibir información suficiente, clara, oportuna y veraz, misma que tendrá como objetivo explicar cuáles son los riesgos y alternativas de los procedimientos diagnósticos, terapéuticos y quirúrgicos que se utilizarán en el tratamiento del paciente, para que pueda decidir libremente su intervención, y pueda otorgar su consentimiento informado, teniendo la opción rechazar tratamientos o procedimientos que considere pertinente. (LEY GENERAL DE SALUD, 2018: art. 77)

Un buen médico tiene la responsabilidad de aplicar sus conocimientos, habilidades y destreza, así como adaptarlos y actualizarlos según los avances técnicos y científicos que la profesión requiera, Es importante mencionar que el médico que realiza un proceso de atencion para la salud siguiendo los lineamientos de la Lex Artis y la ética médica, le va a permitir establecer un vínculo de confianza en la relación entre el médico y el paciente, lo que dará origen a una atención asertiva. (Nicolás Bañuelos, Metabase)

2. Norma Oficial Mexicana NOM-004-SSA3-2012, del expediente clínico La responsabilidad civil extracontractual médico - sanitaria, expresa que la obligación de los médicos y las instituciones de atencion para la salud es de medios y no de resultados con el fundamento de que sus funciones consiste en brindar el servicio en el cual tendrán el compromiso de realizarlo con todas las conductas pertinentes para que se logré su objetivo como lo determina la experiencia de la Lex Artis.

Por consiguiente es la Norma Oficial Mexicana la que regulará los elementos y las condiciones que se deberán cumplir para la integración del expediente clínico, desde luego no incluye de forma automática que se acredite una conducta culposa, ni tampoco acredita de manera obligatoria que se determine la responsabilidad del profesional de la salud, es decir que este tipo de actividades se acreditarán cuando los elementos característicos de la responsabilidad civil concurran, destacando la comprobación de la culpa y su relación con el daño. (TESIS: 2012113. 1a. CXCVIII/2016,10a., 2016)

De ello resulta que, cuando el profesional de la salud tenga una actuación diligente puede realizarse con independencia si el expediente clínico se ha realizado con una buena o mala integración del expediente clínico. En otras palabras, pueden existir casos de atencion médica donde el expediente clínico se haya integrado cumpliendo con todos los requisitos que se manifiestan en la Norma Oficial Mexicana, sin embargo se puede generar una conducta negligente en la atención médica.

En lo que concierne al acto médico es un proceso complejo el cual se debe examinar de forma conjunta 
por estar constituido por sus tres etapas, las cuales son: diagnostica, terapéutica y recuperatoria, está claro que la elaboración del expediente clínico es parte integral del acto médico, por ello está vinculado con todas las actividades que tengan relación con la atencion del paciente, el realizar incumplimiento de los requisitos, elementos o pautas que se determinan en la Norma Oficial Mexicana aplicable no genera de forma obligatoria que la conducta del médico se vuelva dolosa o negligente, ya que su contenido depende de dicha norma, en cambio la regulación del acto médico es parte integrante de la Lex Artis ad hoc. (TESIS: 2012113. 1a. CXCVIII/2016,10a., 2016)

Es por eso que cuando se advierta que existe un expediente clinico incompleto o que se encuentra mal integrado, se considerarán las demás pruebas que se presenten en el juicio y haciendo valer la carga de la prueba que corresponde a cada parte, será el juez quien debe analizar las faltas y elementos incumplidos de la Norma Oficial Mexicana del expediente clínico, para realizar un analisis detallado de la conformación e integración del expediente clinico, con el único objetivo de constatar si la falta del acatamiento o indebido cumplimiento es el acto o una parte del acto, o de lo contrario si la omisión que genero el daño o si a partir de ese acontecimiento se generó una ausencia de la informacion que ha ocasionado la perdida de la oportunidad de poder corregir el daño o impedir que se produjera.(TESIS: 1ª.XXIV/2013,10ª núm. 2002440, 2013)

\section{Lex artis ad hoc}

Actualmente la Lex artis se considera como un término que define el correcto desempeño de quienes se dedican a la profesión médica, teniendo cobertura en diferentes modalidades. Se concibe a la Lex artis como la observación de las reglas generadas por la misma profesión, por ello cuando se utiliza el término Lex artis se refiere a las reglas que determina la profesión para poder realizar su práctica, sin embargo al agregar el vocablo ad hoc se refiere que se sujetaran las actividades profesionales a las circunstancias específicas del caso.

Cuando la doctrina y la jurisprudencia hacen uso del término Lex artis lo realizan entendiéndolo como un saber que tendrá utilidad, correspondiendo a aquellos conocimientos que se supone deben regir las conductas de los profesionales que cuenten con un título, puesto que quienes una profesión tienen la presunción de conocimientos frente a la sociedad, tiene libertad de ejercicio y de decisión, pero también tiene que asumir las responsabilidades que se generen en la práctica profesional. (Teresa Candela, UNAM) 


\section{LA IMPORTANCIA DE LA OBLIGATORIEDAD DEL CONSENTIMIENTO INFORMADO PARA SER PARTE EN LA HISTORIA CLÍNICA}

\section{El acto médico}

\section{$\underline{\text { Concepto }}$}

El acto médico como concepto y categoría sustenta su importancia en que se le considera como el objeto primario para el analisis de la relación que debe existir entre el médico y el paciente. Siendo a través del acto médico que se definen las relaciones juridicas que se pueda generar entre el paciente y los profesionales de la salud, en otras palabras de manera general se define el acto médico como aquel conjunto de acciones que realiza el profesional de la salud que tendrá la recepción de un usuario o paciente en los diferentes servicios de atencion a la salud, que tendrá el objetivo de lograr la recuperación de su salud.(María del Rosario Huerta, UNAM)

\section{Elementos que distinguen y caracterizan al acto médico}

\section{a. Profesionalidad}

Este principio tiene como objetivo primordial la atención médica, la cual únicamente podrá ser efectuada por las personas que ostenten los conocimientos requeridos para ejercer la profesión que acrediten. Dicho principio está regulado en la Ley General de Salud en su artículo 79, mismo que su fundamento se expresa en el artículo 5․ De nuestra Constitución. (Rodrigo Montes.)

b. Lex Artis médica ad hoc

Otro elemento que es de gran importancia para que se genere un buen acto médico es la propia Lex artis al realizar las actividades médicas, esa establece el criterio comprendido para que se pueda determinar si existe o no una responsabilidad médica, es por ello que en el punto central de la argumentación y medio probatorio de los juicios de responsabilidad médica se ubica la pregunta de si hubo cumplimiento o incumplimiento de la Lex artis, este tipo representa a la ejecución del acto médico en el marco de los criterios y forma de actuar aprobados para un determinado tiempo y lugar, en otras palabras, en una situación históricamente concreta como lo es la Lex artis ad hoc. Entendiéndose de esta manera se comprende su precisa y natural relación con la continuidad de la preparación educativa de la medicina. (Manuel Fernando Méndez, 2015)

c. Objetividad

Es por medio del acto médico que se realizan los objetivos de promover la salud, prevenir la enfermedad, 
curarla cuando esté presente y en cuanto sea posible, así como rehabilitar al paciente para que se reintegre a sus actividades acostumbradas. En cambio si no pudiese cumplir con los objetivos que expresa el acto médico, será el profesional de la salud quien tiene la obligación de confortar al paciente, debiendo proporcionarle los medios que se encuentren a su alcance para que logre una mejor calidad de vida en sus posibilidades, incluso el poder llegar a la muerte, de una manera digna para el paciente. (Jaime Cervantes, 2012: p167)

d. Licitud

La doctrina ha expresado que la licitud de la atención médica es aquella que se realice de acuerdo a la Lex artis ad hoc, así como también apegada a las normas correspondiente y que se haya obtenido de parte del paciente su consentimiento o de parte de su representante legal. En México dentro de nuestro sistema juridico encontramos diferentes disposiciones como las siguientes: Ley General de Salud, los reglamentos sanitarios, Las Normas Oficiales Mexicanas, la guías y protocolos de prácticas clínicas que se han emitido por las autoridades competentes que determinan la licitud de la atencion médica. (Jaime Cervantes, 2012: p168)

e. Conducta ética

Se le ha definido cono una ciencia que tiene como objetivo el estudio de lo bueno, lo moral y lo justo, para ser aplicado en el área de la medicina se realiza desde sus inicios relacionando lo bueno con el orden natural, así como lo malo con relación a todo lo que esté en su contra. Siendo la prioridad del médico la de utilizar todos sus conocimientos y experiencias al atender al paciente para que restablezca su salud. (Jaime Cervantes, 2012: p168)

f. No formalidad

En lo que concierne a la no formalidad se refiere que no se necesita que el contrato sea de forma escrita para que se puedan contratar el servicio de atencion médica. Lo anterior no podrá ocasionar algún daño relacionado con la documentación del acto médico, mismo que es obligatorio en el expediente clínico, siempre que esté en relación con los términos previstos en el Reglamento de la Ley General de Salud en materia de prestación de servicios de atencion médica y también en la Norma Oficial Mexicana NOM-168-SSA1-1998, del expediente clínico. (Régimen jurídico del acto médico, CONAMED)

\section{El acto médico documental}

Para realizar en acto médico existen dos formas:

1.- El acto médico directo: Se manifiesta cuando existe contacto directo con el paciente, los cuales se realizan mediante la intervención médica que tendrá como objetivo el obtener la curación o el alivio del enfermo siendo las siguientes: preventivas, diagnosticas o terapéuticas o de rehabilitación. 
2.- El acto médico indirecto: Son actos que no están en contacto con el paciente, en otras palabras son extracorpóreos, se llevan a efecto para mejorar la calidad de la atención médica que solicita el paciente son utilizados para la investigacion, experimentación, enseñanza, administrativa, etc. (Régimen jurídico del acto médico, CONAMED)

Es un hecho que se complementa con los actos descritos anteriormente, por eso su importancia reside en la validez y fuerza como prueba al interior de algún proceso, causa, requerimiento o demanda. En el interior del acto médico documental debe contenerse el certificado médico, la receta o formula médica, así como el expediente clínico, el cual es el más importante de todos.

El expediente clinico ha llegado a adquirir gran importancia, ya que puede realizar el cumplimiento de uno o varios de los siguientes objetivos.

1.- Clínico o asistencial, 2.- Docente o académico, 3.- Investigación, 4.- Control de calidad, 5.Administrativo, 6.- Médico legal. (Régimen jurídico del acto médico, CONAMED)

\section{Médico legal}

Es en el acto médico legal donde se originan los hechos como la justificación de los procedimientos, los exámenes practicados estos pueden ser clínicos y de laboratorio, así como la responsabilidad que tienen los pacientes como la de aquellos que intervienen en la atencion medica, es importante mencionar que en el acto médico legal se encuentran todas las pruebas escritas de cómo se han realizado los tratamientos junto con las reacciones del paciente. Cuando el médico actúa y manipula técnicas y conocimientos con un objetivo a obtener un resultado concreto, este objetivo anhelado por el ejercicio de la medicina sobrelleva responsabilidades, por eso, tiene importancias ante la ley y el expediente clínico va poder permitir que se determine la legalidad del acto médico.

Por esta razón, el acto médico es una fuente donde se generan los resultados jurídicos para el médico que lleva a efecto la atencion médica y para el paciente que está recibiendo la atencion. (Régimen jurídico del acto médico, CONAMED)

\section{El consentimiento informado como cumplimiento de la Lex artis ad hoc con carácter jurídico}

A. Concepto 
En lo concerniente al concepto del consentimiento informado la Ley General de Salud ha expresado que los usuarios de la atencion médica tienen el derecho de recibir la informacion de manera suficiente, que sea clara, oportuna y veraz, y, además que se le oriente con referente a los tratamientos que serán los más adecuados para mejorar su salud, así como también de aquellos riesgos y procedimientos alternativos, diagnósticos terapéuticos y quirúrgicos que se desean realizar. (Ley general de salud, artículo 51 Bis 1.)

El Poder Judicial de la Federación ha sostenido que el consentimiento informado es un resultado necesario o explicación de derechos a la vida, a la integridad Física y a la libertad de conciencia, consistente en un derecho para los pacientes el cual deben otorgar o negarse, siempre y cuando estén debidamente informados de las consecuencias al realizarse los tratamientos o procedimientos médicos, con la finalidad de que se pueda intervenir al paciente, pero previamente a la realización de estos procedimientos es necesario que se le den a conocer las características y las formas de realizar el procedimiento médico, incluyendo los riesgos que se prevén en la intervención. Tal forma que el paciente conoce los riesgos y las consecuencias a las que se puede exponer con la intervención que está legalmente autorizada, sin embargo es importante mencionar que la firma o autorización del consentimiento informado no le resta responsabilidad al médico o institución en caso de que alguno de ellos tenga un actuar negligente. (TESIS: 1a.XLIII/2012, Décima Época, núm. 2001271, 2012)

Conviene subrayar la importancia que tiene el objeto del conocimiento informado, el cual se refiere al tratamiento médico quirúrgico ajustado a la Lex artis ad hoc, Pero esto no alcanza un resultado que sea aleatorio, (Mayra Acevedo, 2017) es decir que exista duda de que pueda o no suceder por existir varios, dado los acontecimiento que se puedan producir en el tratamiento por la existencia de múltiples elementos internos y externos que se puedan generar sin la voluntad del médico que lleva el tratamiento y pueden afectar el objetivo, por ser la obligación de medios o actividad que dirige su actuación, aunque la diligencia que se exige sea la propia de las obligaciones de realizar el mayor esfuerzo, ante la trascendencia vital que, en muchas ocasiones, protege al paciente la actuación del médico.(Mayra Acevedo, 2017)

B. La carga probatoria y las consecuencias juridicas por el incumplimiento del deber de informar por el médico.

Cuando un médico se encuentre demandado por responsabilidad civil extracontractual que se encuentre relacionada con la negligencia médica, donde la ausencia del cumplimiento del deber de informar sea la causa de un hecho negativo, porque se argumente que no se le informo al paciente o que la informacion proporcionada fue deficiente, al médico demandado le corresponde la obligación de la carga de la prueba, para demostrar que sí se realizó dicha obligación que recae al médico demandado. (Código de Procedimientos Civiles para el Distrito Federal, 2015, arts. 281 y 281) 
Los daños y perjuicios que se ocasionen a tercero por comparecer, o exhibir cosas, serán indemnizados por la parte que ofreció la prueba, o por ambas si el juez procedió de oficio, sin perjuicio de hacer la regulación de costas en su oportunidad. Las partes asumirán la carga de la prueba de los hechos constitutivos de sus pretensiones.

De ello resulta, que cuando un médico que haya tratado al paciente niegue haber faltado con su deber de informar, evidentemente estará confirmando un hecho fundamentado en que otorgó la informacion necesaria de forma más adecuada, teniendo como consecuencia la finalidad de comprobar dicha afirmación, pudiendo aportar todos aquellos medios que se encuentren a su alcance, mismos que le serán más fácilmente obtener por contar con los conocimientos propios especializados de la materia, así como testimonios de otros médicos documentales privadas como lo es el expediente clinico, en el cual se registra la comunicación que debe existir con el paciente o con sus familiares, tutores o representantes, etc. (TESIS: 1ª. CCXXVI/2016, 10ª núm. 2012512, 2016)

En caso que no se llegase a demostrar que el o los profesionales de la salud cumplieron con el deber de informar, es motivo para que se actualice un acto negligente en una evidente desobediencia a la Lex artis ad hoc que satisface uno de los elementos de la acción de responsabilidad civil extracontractual. Sin embargo, el hecho de que exista una negligencia médica por falta al deber de informar, no se genera en inmediatamente una responsabilidad civil, está claro que se tiene que demostrar, coincidentemente, la existencia de un daño, siendo ese acto el que lo genero de manera negligente o fue como un elemento determinante en la realización del daño. (TESIS: 1ª. CCXXVI/2016, 10ª núm. 2012512, 2016)

\section{EL CONCEPTO DE LA PRUEBA Y SU VINCULACION CON LA HISTORIA CLÍNICA EN EL PROCESO CIVIL}

\section{Definición de prueba}

¿Qué es la prueba?

(Ovalle Fabela, 2016, p. 332.) Explica que: será prueba en sentido estricto la obtención de la certeza del juzgador sobre los hechos cuyo esclarecimiento es necesario para la resolución del conflicto sometido a proceso. En este sentido, la prueba es la verificación o confirmación de las afirmaciones de hecho expresada por las partes.

Por lo que se refiere al Código Federal de Procedimientos Civiles manifiesta que para conocer la verdad, el juez debe conocer los hechos para ello podrá ayudarse de cualquier persona que sea parte o tercero, así como también podrá apoyarse de cualquier objeto o documento, que sea propiedad de alguna de las partes o de un tercero, sin tener ningún impedimento será suficiente que estén reconocidas por la ley y estén relacionadas de 
manera inmediata con los hechos discutidos. (Código Federal de Procedimientos Civiles, 2012, art. 79)

Por consiguiente, para acreditar la responsabilidad civil de los médicos, cuando se encuentren demandados por que se les atribuye un daño, serán ellos quienes tengan que probar su debida diligencia.

No podemos olvidar que el expediente clinico es el documento que se utiliza como prueba documental en caso de alguna inconformidad, queja o demanda en contra de los servicios médicos y por lo tanto el expediente clinico es determinante para acreditar la diligencia o la mala práctica del médico en el proceso a la atencion de la salud.

\section{La prueba documental}

Cualquier cosa que haya sido producido por la actividad humana, que pueda ser apreciable con los sentidos de la vista y el tacto, será considerado como un documento, el cual va a servir como prueba histórica indirecta y representativa de un hecho cualquiera.

Sera de tipo declarativo - representativo si en el contenido existe una declaracion de parte de quien lo ha creado, lo ha otorgado o simplemente si alguien lo ha firmado, tal como lo son los escritos púbicos o privados, sin embargo pueden ser solamente representativo, en el caso que no contenga ninguna declaracion por ejemplo los planos, cuadros o fotografía, de ello resulta que el documento no siempre tendrá la característica de ser escrito.

Su raíz etimológica confirma su carácter representativo, dado que la voz documento deriva del vocablo docere que tiene el significado de enseñar o hacer conocer. Por lo tanto la prueba documental es el medio de convicción mediante el cual una de las partes que este en el litigio se servirá para poder demostrar un hecho que se encuentre relacionado a los argumentos discutidos en el procedimiento de referencia. (TESIS: 184814. I.14o.C.4 K, 9 época, 2003.)

Sobre las bases de las líneas anteriores podemos determinar que la historia clínica es una prueba documental de los dos tipos.

Es declarativa - representativa porque en ella se manifiesta el consentimiento informado que es la expresión de la voluntad del paciente de aceptar los procedimientos médicos mismo que en él se plasma su firma, con referente al médico tratante, se manifiesta la informacion que le ha proporcionado al paciente, la cual también es firmada, la institución médica o el prestador del servicio médico que genere la historia clínica serán los propietarios, cuando no dependan de una institución, sin embargo las instituciones del sector público, se regirán con lo establecido en la Norma Oficial Mexicana NOM-004-SSA3-2012, Del expediente clínico.

Así mismo es representativa porque en su contenido se encuentran elementos de tipo gráficos, imagenológicos, electrónicos, magnéticos, electromagnéticos, ópticos, magneto-ópticos, así como aquellos que se 
realicen con otras tecnologías, que den testimonio de la atencion médica que ha recibido el paciente.

\section{Objeto de la prueba}

¿Qué se prueba?

En el proceso civil se busca demostrar los hechos afirmados y discutidos por las partes, por consiguiente con la historia clinica como prueba documental se tendrá como objetivo probar la mala práctica médica producida por los profesionales de la salud cuando cometen impericia, negligencia o imprudencia por incumplir las obligaciones que tienen los profesionales de la salud de proporcionar al paciente los cuidados que sean necesarios para restablecer su salud, utilizando sus conocimientos profesionales, así como los técnicos y científicos siempre bajo el criterio de la pericia y prudencia para evitar ser responsable de una consecuencia trágica de la enfermedad que padece el paciente o por no curar la enfermedad, incluso por llegarse a producir el fallecimiento del paciente. (Código Federal de Procedimientos Civiles, 2012)

\section{Carga de la prueba}

\section{¿Quién prueba?}

En el momento que una persona argumente que un profesional médico-sanitario o una institución hospitalaria le ha generado un daño por una inadecuada atencion del servicio médico, se renueva lo que se nombra como una reinversión de la carga de la prueba a favor de la parte actora en el juicio, donde el médico o la institución hospitalaria serán quienes tengan que acreditar la debida diligencia que se realizó en la atencion médica del paciente que sufrido el daño, bajo los principios de facilidad y proximidad probatoria.

El objetivo es buscar que ambas partes participen enérgicamente en el proceso y que aporten las pruebas de convencimiento que sean necesarias para que el juez llegue a la verdad y poder realizar un estudio para acreditar o no los elementos de la acción. (TESIS: 2012513. 1a. CCXXVII/2016, 10a, 2016)

En Mexico para el proceso civil existen dos reglas elementales para la distribución de la carga de la prueba. La primera será la parte actora quien tenga que probar los motivos de hecho de su pretensión en cambio la parte demandada debe probar los hechos de su excepción o defensa. La segunda regla expresa que solo el que afirma tendrá la carga de la prueba de sus afirmaciones de los hechos, así como aquel que niega, solo debe probar en los casos excepcionales que así o considere la ley. (Ovalle Fabela, 2016, p. 332.) 


\section{Procedimiento probatório}

¿Cómo se prueba?

Será a través del procedimiento probatorio donde se presentara la historia clinica como prueba, la cual tendrá que desarrollar fundamentalmente los siguientes actos.

1.- el ofrecimiento o proposición de los medios de prueba, que corresponden a las partes.

2.- La admisión o el desechamiento de los medios de prueba, que lleva a cabo el juzgador, tomando en cuenta básicamente la idoneidad o la falta de aptitud de los medios de prueba para acreditar los hechos y la relevancia de estos para la decisión del litigio.

\section{3.- Preparación de los medios admitidos.}

4.- La ejecución, práctica o desahogo de las pruebas admitidas y preparadas, la cual se lleva a cabo en la audiencia correspondiente.

5.- La valoración o apreciación de las pruebas practicadas, que realiza el juzgador en la sentencia. (Ovalle Fabela, 2016, p. 332.)

5. Valoración de la prueba

¿Qué valor tiene la prueba producida?

Sin embargo los tribunales solo aceptarán como medios de prueba aquellos que estén autorizados por la ley, la cual no le otorga el mismo valor probatorio a dichas pruebas, es decir que se establecen categorías entre los medios de prueba, de esta manera tenemos que hay prueba plena y otras que carecen de ese valor, las cuales son simples indicios que solo el juez podrá tener el criterio para tomar la decisión de aceptar o no.

El Código Federal de Procedimientos Civiles reconoce que los documentos publicos hacen prueba plena de los hechos cuando están legalmente firmados por la autoridad que los ha originado, por el contrario, si en sus contenido existen declaraciones de verdad o exposiciones de hechos particulares, por consiguiente estos documentos solo van a probar plenamente que en presencia de ellos se originaron las declaraciones o manifestaciones, por consiguiente no van a probar la verdad de lo manifestado en las declaraciones. (Código Federal de Procedimientos Civiles, 2012, arts.197 y202)

En relación con los documentos privados, estos forman prueba de los hechos expresados en el documento, siempre y cuando sean contrarios a los intereses de quien los haya generado o en casos donde la ley no disponga de otra cosa. Cuando el documento sea originado por un tercero únicamente va ser considerado como prueba en favor de la parte que desea beneficiarse con él, y se prueba en contra para su colitigante, siempre y cuando no lo impugne.

Referente a los medios electrónicos, ópticos o cualquier otro generado por tecnología serán reconocidas 
como pruebas la informacion que estos medios generen.

De esta manera en el procedimiento civil se reconoce como medios de prueba: La confesión, los documentos públicos, los documentos privados, los dictámenes privados, los dictámenes periciales, el reconocimiento o inspección judicial, los testigos, las fotografías, escritos y notas taquigráficas y de manera general todos aquellos elementos que se aporten por los descubrimientos de la ciencia y las presunciones. (Código Federal de Procedimientos Civiles, 2012, arts.203, 210 y 93 )

Dicha valoración de la prueba se realiza en el momento en que el juez dicte la sentencia, siendo en este momento cuando se fija los hechos controvertidos tomando como base las pruebas rendidas y serán los hechos demostrados los que

Establecen el supuesto factico de la norma jurídica que se deberá aplicar en el caso discutido. (Orizaba Monroy, Salvador, 2013: pp. 171 y 172.)

Por consiguiente podemos exponer que la historia clinica es una prueba plena al contener elementos característicos de un documento público, por contar con la firma del médico que ha generado la historia clinica, en ella se encuentra los comentarios fidedignos que el paciente ha manifestado con relación a sus padecimientos, así como los hechos que han originado los padecimientos, pero hay que dejar claro que estos documentos solo van a probar plenamente que en presencia de quien se ha generado la historia clinica se originaron las declaraciones o manifestaciones del paciente, por tal motivo no van a probar la verdad de lo manifestado en las declaraciones.

Por otra parte la historia clinica cuenta también con caracteristicas de un documento privado, en base a que los datos de su contenido forman prueba de los hechos relatados en ella, hay que aclara que siempre y cuando sean contrarios a los intereses del médico o que la ley disponga otras indicaciones. Sin embargo cuando la historia clinica sea realizada por una tercera persona únicamente se va a considerar como una prueba que estará a favor de quien desea obtener beneficio del contenido, consolidándose como una prueba en contra de la otra parte del conflicto, siempre y cuando no se inconforme con la prueba.

Al mismo tiempo la historia clinica está conformada por los contenidos en otros documentos ya sean escritos, gráficos, imagenológicos, electrónicos, magnéticos, electromagnéticos, ópticos, magneto-ópticos, así como aquellos que se realicen con otras tecnologías, en las cuales mediantes ellas se hagan constar la atención médica en los diferentes momentos de su procedimiento, mismos que también son reconocidos como pruebas.

\section{Medio de prueba}

¿Cómo se prueba?

En el proceso civil se va a probar con los instrumentos y aquellas conductas humanas, por medio de las 
cuales se pretende lograr que se verifiquen las afirmaciones de los hechos. Por esta razón para determinar la responsabilidad civil del médico que realizó la atención médica se realizará con nuestro instrumento probatorio que es la historia clínica. (Orizaba Monroy, Salvador, 2013: pp. 171 y 172.)

\section{CONCLUSIONES}

No cumplir con lo que se ha establecido en la Norma Oficial Mexicana del expediente clinico, no va a generar de manera inmediata la confirmación de que se realizó una conducta dolosa y por consiguiente tampoco involucra de manera obligatoria la responsabilidad de aquellos que realizan la atencion medica, está claro que primero hay que comprobar que se tienen manifestados los elementos de la responsabilidad civil.

Se deberá analizar de forma conjunta el acto médico, es decir todas las etapas que lo integran desde la diagnostica, terapéutica y recuperadora, el no cumplir con alguno de los requisitos del acto médico no genera que la conducta del médico sea dolosa o negligente, es por ello que para determinar la responsabilidad médica se tiene que analizar la manifestación de la norma mexicana en conjunto con la regulación del acto médico ya que este forma parte de la Lex artis ad hoc. Evaluar el acto médico en forma sectorizada sin tomar en cuenta todas las etapas que lo integran será inadecuado e ilógico ya que las etapas del acto médico están inmersas en una secuencia de tiempo.

Es el consentimiento informado la manera de comprobar la legitimidad del acto médico, en él se encuentra el derecho que tiene el paciente de su autonomía o autodeterminación, definitivamente es una exigencia ética y legal que debe obedecer el médico, pero, también es parte del acto clinico y su importancia está bajo la normativa de la lex artis y su incumplimiento puede ocasionar responsabilidad.

Es en el juicio ordinario civil donde será posible demandar al médico en lo particular o a una sociedad privada que se a la que proporciono el servicio de atencion medica. La responsabilidad civil que se le imputa al médico o a la institución tiene como fundamento el daño que se le ha ocasionado al paciente, el cual podría generar una responsabilidad de tipo subjetiva, para la cual es necesario que se demuestre la culpa o negligencia del personal médico responsable.

Para eso es el juez quien debe especificar cuáles son los deberes de los médicos en cada caso concreto; es decir, no obstante que la lex artis puede tener un elemento efectivo, pues se conforma por la práctica médica, es el juzgador quien debe determinarla a la luz del material probatorio del que disponga o del que pueda allegarse.

Por todo lo anterior se recomienda capacitar al personal de salud de las instituciones públicas o privadas en el tema referente a la normatividad del expediente clinico, así como también informarlo de los alcances jurídicos que se harán acreedores cuando cometan un incumplimiento en el acto médico y las posibles 
responsabilidades civiles que se generarían por su actuación.

Realizar de manera frecuente revisiones y actualizaciones de los formatos con los que se elabora el expediente clinico verificando que se realicen con apego a la normatividad y su aplicación práctica.

Fomentar la práctica de auditorías médicas como un proceso interdisciplinario lo cual va a permitir que el cuerpo médico pueda realizar evaluaciones del acto médico, con los objetivos de mejorar la práctica médica, ser un mejor medio de formación académica y lo más importante evaluar para obtener una mejor calidad de la atencion médica en México.

\title{
"CLINICAL HISTORY AS PROOF FOR CIVIL RESPONSIBILITY FOR BAD MEDICAL PRACTICE"
}

\begin{abstract}
To accredit the civil liability of doctors, when they are sued because they are attributed damage, the medical act becomes very important. It is considered as the legal instrument where the doctor's performance is shown, constituting as the documentary evidence of greater legal weight in case of claims of a civil nature. For the development of the topic we will consider as a hypothesis the analysis of the stages of the medical act in a continuous way, it will allow to know the characteristic elements of the clinical history that have not been fulfilled in the lex artis ad hoc, to determine the civil responsibility of the doctor when being used as documentary proof. Using for the development, the contemporary methods of investigation as they are it the analytical doctrine and the legal sociology. The foregoing justifies an analysis of legal doctrine and regulations to be considered as evidence in the process of medical civil liability..
\end{abstract}

Keywords: Medical act, Clinical record, Medical liability, Lex artis ad hoc, Civil Process 


\section{BIBLIOGRAFÍA}

\section{Doctrina}

Acevedo Camargo, Mayra Guadalupe, (2017) Regulación en México del consentimiento informado, (eds.) Biblioteca Virtual del Instituto De Investigaciones Jurídicas de la UNAM, México.

Alcázar Agüero, Maritza, Nápoles Ramón, Yaimí, et alt, (2010) “La historia clínica: un documento básico para el personal médico", Revista MEDISAN, vol. 14, núm. 7 Santiago de Cuba.

Cervantes Martínez, Jaime Daniel,(2010) Responsabilidad civil médica: el derecho a la salud y el médico, (eds.) LIBMAR, México.

Galván Meléndez, Manuel Fernando, González Hernández, José Agustín, et. alt .(2015) "Responsabilidad profesional en el ejercicio de la medicina", Revista Médica, año 7, núm. 1, México.

Germán Fajardo, Dolci., y Hernández Torres, Francisco, (2012), (eds.) Definiciones y conceptos fundamentales para el mejoramiento de la calidad de la atencion a la salud, Secretaría de Salud, México.

Huerta Lara, María del Rosario, (2015) El acto médico y sus implicaciones en el derecho civil, (eds.) Letras Jurídicas, Instituto de Investigaciones Juridicas, Universidad de Veracruz, letrasjuridicas.com.mx/Volumenes/32/A04.pdf

LINEAMIENTOS PARA EL MANEJO DEL EXPEDIENTE CLÍNICO, (2014) (eds.)Secretaría de Salud, Hospital Juárez de México.

López López, Fidel Herminio, Ortega González, Manuel, et alt, (2009) “La importancia del expediente clínico como prueba documental”, Revista de Evidencia e Investigación clínica, Vol. 2, Núm. 2.

López López, Fidel Herminio, Ortega González, Manuel, y Portillo Mijangos, Rubén Celestino,(2010) El ejercicio de la medicina apegada al derecho médico, (eds.) THEMIS, México. 
Ornelas Aguirre, José Manuel, (2013) El expediente clínico, (eds.) El Manual Moderno, Universidad de Sonora, México.

Ovalle Favela, José, (2016) Teoria general del proceso, 7a. Edición, (eds.)OXFORD, México.

\section{Legislacion Nacional}

CÓDIGO FEDERAL DE PROCEDIMIENTOS CIVILES,(2012) Nuevo Código publicado en el Diario Oficial de la Federación el 24 de febrero de 1943, Texto Vigente, Última reforma publicada DOF.

CÓDIGO DE PROCEDIMIENTOS CIVILES PARA EL DISTRITO FEDERAL, (2015), Código publicado en el Diario Oficial de la Federación los días 1o. al 21 de septiembre de 1932. Última reforma publicada en la Gaceta Oficial del Distrito Federal de fecha 2 de junio.

LEY GENERAL DE SALUD, ( 2018) Nueva Ley publicada en el Diario Oficial de la Federación el 7 de febrero de 1984, TEXTO VIGENTE, Última reforma publicada DOF 11-05.

NORMA OFICIAL MEXICANA NOM-004-SSA3-2012, Del expediente clínico. dof.gob.mx/nota_detalle.php?codigo=5272787, (consultada: 20 de junio, 2018)

NORMA OFICIAL MEXICANA NOM-040-SSA2-2004, En materia de información en salud.

\section{Tesis y resoluciones de la corte}

TESIS: 2002569. 1a. XXVIII/2013 (10a.). Primera Sala. Décima Época. Semanario Judicial de la Federación y su Gaceta. Libro XVI, enero de 2013, Pág. 638, MALA PRÁCTICA MÉDICA. AUSENCIA O DEFICIENCIA DE LA HISTORIA CLÍNICA.

TESIS: 2004786. I.4o.A.91 A (10a.). Tribunales Colegiados de Circuito. Décima Época. Semanario Judicial de la Federación y su Gaceta. Libro XXV, octubre de 2013, Pág. 1891.RESPONSABILIDAD PROFESIONAL MÉDICA. SIGNIFICADO DEL CONCEPTO LEX ARTIS PARA EFECTOS DE SU ACREDITACIÓN. 
TESIS: 1ª.XXVIII/2013 (10ª), Aislada Civil, núm. 2002569, Décima Época, Semanario Judicial de la Federación y su Gaceta, Libro XVI, Tomo I, enero 2013. pág. 638. MALA PRÁCTICA MÉDICA. AUSENCIA O DEFICIENCIA DE LA HISTORIA CLÍNICA.

TESIS: 1ª. CCXXVII72016, (10a), Aislada Civil, núm. 2012513, Décima Época, Semanario Judicial de la Federación y su Gaceta, Libro 34, Tomo I, septiembre de 2016, pág. 514, RESPONSABILIDAD CIVIL EXTRACONTRACTUAL EN MATERIA MÉDICO- SANITARIA. DISTRIBUCION DE LA CARGA DE LA PRUEBA.

TESIS: 2012113. 1a. CXCVIII/2016 (10a.). Primera Sala. Décima Época. Gaceta del Semanario Judicial de la Federación. Libro 32, Julio de 2016, Pág. 324, RESPONSABILIDAD CIVIL EXTRACONTRACTUAL POR NEGLIGENCIA MÉDICA. ESTÁNDAR PARA VALORAR SI EL INCUMPLIMIENTO DE LA NORMATIVA QUE REGULA UN EXPEDIENTE CLÍNICO ACTUALIZA O NO UNA CONDUCTA NEGLIGENTE.

TESIS: 1ª XXIV/2013 (10a.), Tesis Aislada Civil, Décima Época, núm. 2002440, Semanario Judicial de la Federación y su Gaceta, Libro XVI, Tomo 1, enero de 2013, pág. 621, ACTO MÉDICO. DISTINTAS ETAPAS O FASES QUE LO CONFORMAN PARA EFECTOS DE DETERMINAR LA EXISTENCIA DE UNA POSIBLE MALA PRÁCTICA MÉDICA.

TESIS: 1a. XLIII/2012, Décima Época, Semanario Judicial de la Federación y su Gaceta, núm. 2001271, Libro XI, agosto de 2012, Tomo 1, p. 478, rubro: INFORMADO. DERECHO FUNDAMENTAL DE LOS PACIENTES.

TESIS: 1a. CCXXVI/2016 (10a.), Tesis Aislada Civil, Décima Época, Gaceta del Seminario Judicial de la Federación, núm. 2012512, Libro 34, Tomo I, septiembre 2016, pág. 513, RESPONSABILIDAD CIVIL EXTRACONTRACTUAL. CARGA PROBATORIA Y CONSECUENCIAS POR EL INCUMPLIMIENTO DEL DEBER DE INFORMAR EN MATERIA MÉDICO-SANITARIA.

TESIS: 184814. I.14o.C.4 K. Tribunales Colegiados de Circuito. Novena Época. Semanario Judicial de la Federación y su Gaceta. Tomo XVII, Febrero de 2003, Pág. 1118.PRUEBA DOCUMENTAL CONCEPTO. TESIS: 2012513. 1a. CCXXVII/2016 (10a.). Primera Sala. Décima Época. Gaceta del Semanario Judicial de la 
Federación. Libro 34, Septiembre de 2016, Pág. 514, RESPONSABILIDAD CIVIL EXTRACONTRACTUAL EN MATERIA MÉDICOSANITARIA. DISTRIBUCIÓN DE LA CARGA DE LA PRUEBA.

\section{Publicaciones y paginas de internet}

RÉGIMEN JURÍDICO DEL ACTO MÉDICO. www.conamed.gob.mx/prof_salud/pdf/acto_medico.pdf (consultada el 18 de junio de 2018)

Exámenes Paraclínicos - Servicios Médicos Olimpus. www.olimpuslab.com/servicios-estrategicos/medicinadel.../examenes-paraclinicos. (Consultada el 18 de junio de 2018)

Guzmán, Fernando, y Arias, Carlos Alberto, La historia clínica: elemento fundamental del acto médico, www.scielo.org.co/pdf/rcci/v27n1/v27n1a2.pdf, (Consultada el 19 de junio de 2018)

Jiménez Candela, Teresa, Lex artis y responsabilidad médico-sanitaria: una perspectiva actualizada, UNAM, https://archivos.juridicas.unam.mx/www/bjv/libros/4/1943/22.pdf, (Consultada el 19 de junio de 2018)

Montes de Oca Arboleya, Rodrigo, Acto médico y responsabilidad profesional: reflexiones en torno a las diferencias entre las jurisdicciones mexicana y estadounidense, 207.249.17.176/Transparencia/Lists/.../RODRIGO\%20MONTES\%20DE\%20OCA.pdf, (Consultada el 21 de junio de 2018)

Bañuelos Delgado, Nicolás, El expediente clínico, Metabase UAEM, sined.uaem.mx:8080/xmlui/handle/123456789/2569, (Consultada el 21 de junio de 2018)

Trabalho enviado em 11 de julho de 2018

Aceito em 04 de setembro de 2018 